month. Exposures of HAFM cases were compared with those of other patients sampled from the same wards. Nursing practices were observed, and an anonymous questionnaire was issued to assess methods for administration of parenteral drugs.

Of the 21 LAFM cases, 20 (95\%) had a previous hospital admission (exposure admission) compared with 15 (25\%) of 61 other patients $(P<.001)$. During the exposure admission, all HAFM patients had occupied the same room as, or a room adjacent to, an AFM patient; 14 (23\%) of the 60 other patients occupied the same room or rooms adjacent to an AFM patient $(P<.001)$. Ninety percent of the HAFM patients received infusions through a heparin lock during the exposure admission, compared with $49 \%$ of 120 general patients $(P<.001)$. Ten percent of the nurses admitted to using one syringe for more than one heparin lock, and $50 \%$ filled syringes with enough heparin for 3 to 10 heparin locks.

Investigators concluded that $P$ falciparum was transmitted between patients when single syringes were used on heparin locks of sequential patients. They note that nosocomial bloodborne transmission of malaria is less likely to occur than transmission of hepatitis B, HIV, viral hemorrhagic fevers, or other bloodborne agents. To transmit malaria, transfer of intact erythrocytes is needed, thus the link to heparin: the blood must be fresh.

Concern was expressed by the investigators that this practice of using one syringe, with or without a needle, on the intravenous device of more than one patient might be a widespread practice. Nurses and doctors that staff this hospital come from a variety of Asian, Middle Eastern, and African countries. Use of a single syringe for injection into the intravenous lines of multiple patients for administration of anesthesia has led to nosocomial infections in the United States. Fluid in an intravenous device represents a direct conduit to the patient's blood, and only rigid adherence to correct use of devices that access this fluid will prevent transfer of infectious agents.

FROM: Abulrahi HA, Bohlega EA, Fontaine RE, et al. Plasmodium falciparum malaria transmitted in hospital through heparin locks. Lancet 1997;349:23-25.

\section{Nosocomial Infections in High-Risk Nurseries}

The CDC's National Nosocomial Infection Surveillance (NNIS) System recently published data on the epidemiology of nosocomial infections among neonates in 99 hospitals with Level III high-risk nurseries that participate in NNIS. All 99 hospitals use standard surveillance protocols and nosocomial infection site definitions. The data included information on, and risk factors for, infection such as device exposure, birth-weight category $(<1,000 \mathrm{~g}, 1,001 \mathrm{~g}$ through $1,500 \mathrm{~g}$, $1,501 \mathrm{~g}$ through $2,500 \mathrm{~g}$, and $>2,500 \mathrm{~g}$ ), mortality, and the relationship of nosocomial infection to death.

From October 1986 through September 1994, these hospitals submitted data on 13,179 nosocomial infections. The bloodstream was the most frequent site of nosocomial infection in all birth-weight groups. Nosocomial pneumonia was the second most common infection site, followed by the gastrointestinal and eye, ear, nose, and throat sites. The most common nosocomial pathogens among all neonates were coagulase-negative staphylococci, Staphylococcus aureus, enterococci, Enterobacter species, and Escherichia coli. Group B streptococci were associated with $48 \%$ of bloodstream infections that were acquired maternally; coagulase-negative staphylococci were associated with $58 \%$ of the bloodstream infections that were not acquired maternally, most of which (88\%) were associated with umbilical or central intravenous catheters.

The CDC recommends that bloodstream infections, the most frequent nosocomial infections in all birth-weight groups, should be a major focus of surveillance and prevention efforts in high-risk nurseries. Within the nursery, bloodstream infection surveillance should focus on umbilical or central intravenous catheter use, a major risk factor for infections.

FROM: Gaynes RP, Edwards JR, Jarvis WR, et al. Nosocomial infections among neonates in high-risk nurseries in the United States. Pediatrics 1996;98:357-361.

Additional news items in this issue: Dramatic Drop in AIDS Deaths, page 164; Influenza Vaccination of Workers Reduces Mortality in Elderly Patients, page 182; Risk Factors for Nursing Home Outbreaks, page 208; Foege Named Professor, page 214. 